\title{
Characterization, Taxonomy, and Emended Description of Vibrio metschnikovii
}

\author{
J. V. LEE, T. J. DONOVAN, AND A. L. FURNISS \\ Public Health Laboratory, Preston Hall, Maidstone, Kent ME20 7NH, United Kingdom
}

\begin{abstract}
A numerical taxonomic study was carried out to establish: (i) the relationship of freshly isolated, oxidase-negative, vibrio-like organisms to strains listed in certain culture collections as Vibrio metschnikovii and V.proteus; (ii) the relationship of all these oxidase-negative organisms to the genus Vibrio and related organisms. Eighty-three strains were tested for their abilities to grow on 49 compounds as the sole organic source of carbon, their fermentation of carbohydrates, and their production of extracellular enzymes; various physiological tests were also carried out. In all, 130 characters were determined for each strain. The collection of strains were of Vibrio and Aeromonas species, certain other possibly related organisms, and 40 oxidase-negative organisms from a wide range of habitats. It was concluded that all of the oxidase-negative, vibrio-like strains except one belong to the species Vibrio metschnikovii Gamaleia 1888 (synonyms $V$. proteus Buchner 1885; $V$. cholerae biotype proteus Shewan and Veron 1974). The lectotype is NCTC 8443.
\end{abstract}

Certain culture collections contain strains listed as Vibrio proteus (Buchner) and V. metschnikovii (Gamaleia). V. proteus was originally isolated in 1884 from feces of patients suffering from cholera, and V. metschnikovii was isolated from a fowl that had died of a choleraic disease. It has been suggested that these two species be considered synonyms of $V$. cholerae biotype proteus (23). These organisms are unusual for vibrios since they are negative in the Kovács oxidase test (14).

In recent years we have frequently isolated, both from the marine environment and from freshwater, organisms that are vibrio-like and grow on thiosulfate-citrate-bile-salt-sucrose agar (TCBS) but are oxidase negative. We have also received many similar strains for identification from workers throughout the world. These strains are widely distributed both geographically and in their habitat.

The Subcommittee on the Taxonomy of Vibrios of the International Committee on Systematic Bacteriology has pointed out that very little is known about the relationship of these oxidase-negative organisms to the oxidase-positive Vibrio species and that their classification has not been studied in detail (12). To our knowledge no previous taxonomic study has included freshly isolated oxidase-negative strains similar to those described here. We have, therefore, examined the relationships between the wild strains and the culture collection strains. We have also studied the relationships between this oxidase-negative group, the genus Vibrio, and other possibly related organisms. We selected our wild oxidase-negative strains to be as representative as possible of the isolates available to us at the time.

\section{MATERIALS AND METHODS}

Bacterial strains. The cultures used are listed in Table 1. Four strains, NCTC 8443, ATCC 7708, NCTC 8563 , and NCTC 8564 , were tested in duplicate since we had received separate cultures of them direct from the appropriate culture collection and via $\mathrm{R}$. Hugh. These duplicate cultures did not behave identically.

The strains that required sodium for growth were maintained on slopes of marine agar (MA; Difco) at $4^{\circ} \mathrm{C}$, in the dark under sterile liquid paraffin. Other cultures were maintained on slopes of heart infusion agar (Difco) under sterile liquid paraffin, in the dark at room temperature. Unless otherwise stated, strains were incubated at $30^{\circ} \mathrm{C}$ for all tests. $V$. fischeri 62 and Photobacterium phosphoreum 82 grow poorly at $30^{\circ} \mathrm{C}$ and were therefore tested at $20^{\circ} \mathrm{C}$.

Motility and morphology. Motility was determined by the hanging drop method from $1 \%$ tryptone water (Oxoid) containing $2 \%$ (wt/vol) $\mathrm{NaCl}\left(\mathrm{T}_{1} \mathrm{~N}_{2}\right.$ ). Flagella were stained by the Rhodes method (19) as modified by Gauthier et al. (9). A few strains were also examined by electron microscopy.

Salt tolerance. All strains were tested for their ability to grow in $1 \%$ tryptone water containing $0,0.5$, $1,2,3,4,5,6,7,8$, and $10 \%$ (wt/vol) $\mathrm{NaCl}$. Tubes containing $3 \mathrm{ml}$ of medium were inoculated with 10 $\mu \mathrm{l}$ of an early-log-phase $\mathrm{T}_{1} \mathrm{~N}_{2}$ broth culture containing approximately $10^{3}$ to $10^{4}$ colony-forming units per $\mathrm{ml}$. Growth was determined visually after $48 \mathrm{~h}$ of incubation.

Temperature range. Strains were tested for their ability to grow at different temperatures in $T_{1} N_{2}$. The 
TABLE 1. Strains used

\begin{tabular}{|c|c|c|c|c|c|}
\hline \multirow[b]{2}{*}{ Computer no. } & \multirow{2}{*}{ Laboratory no. } & \multicolumn{2}{|c|}{ Received as: } & \multirow{2}{*}{ Source $^{a}$} & \multirow[b]{2}{*}{ Habitat } \\
\hline & & Name & Strain & & \\
\hline \multicolumn{6}{|c|}{ Oxidase-negative vibrios } \\
\hline 1 & MV 409 & V. metschnikovii & NCTC 8443 & (1) & Fowl \\
\hline 2 & MV 501 & $V$. proteus & P 6915 & (2) & \\
\hline 3 & MV 2066 & Original isolate & & & Lobster \\
\hline 4 & MV 2067 & Original isolate & & & Lobster \\
\hline 5 & MV 2068 & $V$. proteus & NCTC 8563 & (1) & \\
\hline 6 & MV 2069 & V. proteus & NCTC 8564 & (1) & \\
\hline 7 & MV 2196 & V. metschnikovii & ATCC 7708 & (3) & \\
\hline 8 & MV 2229 & Vibrio sp. & & (4) & Sewage \\
\hline 9 & MV 2318 & Original isolate & & & Cockles \\
\hline 10 & MV 2338 & Vibrio sp. & & (5) & River water \\
\hline 11 & MV 2339 & Vibrio sp. & & (5) & River water \\
\hline 12 & MV 2345 & Vibrio sp. & & (5) & River water \\
\hline 13 & MV 2346 & Vibrio sp. & & (5) & River water \\
\hline 14 & MV 2360 & Vibrio sp. & & (4) & Sewage \\
\hline 15 & MV 2361 & Vibrio sp. & & (4) & Sewage \\
\hline 16 & MV 2362 & Vibrio sp. & & (4) & Sewage \\
\hline 17 & MV 2365 & Vibrio sp. & & (4) & Sewage \\
\hline 18 & MV 2366 & Vibrio sp. & & (4) & Sewage \\
\hline 19 & MV 2375 & Vibrio sp. & & (4) & Sewage \\
\hline 20 & MV 2376 & Vibrio sp. & & (4) & Sewage \\
\hline 21 & MV 2377 & Vibrio sp. & & (4) & Sewage \\
\hline 22 & MV 2383 & Vibrio sp. & & (5) & River water \\
\hline 23 & MV 2401 & V. metschnikovii & ATCC 7708 & (6) & \\
\hline 24 & MV 2402 & V. metschnikovii & NCTC 8443 & (6) & Fowl \\
\hline 25 & MV 2403 & V. metschnikovii & & (6) & \\
\hline 26 & MV 2404 & $\begin{array}{c}\text { Vibrio sp. (cholera } \\
\text { gp.) }\end{array}$ & ATCC 11554 & (6) & \\
\hline 27 & MV 2406 & $V$. proteus & NCTC 8563 & (6) & \\
\hline 28 & MV 2407 & V.proteus & NCTC 8564 & (6) & \\
\hline 29 & MV 2409 & $V$. tyrogenes & & (6) & \\
\hline 30 & MV 2423 & Vibrio sp. & & (5) & River water \\
\hline 31 & MV 2468 & Original isolate & & & Cockles \\
\hline 32 & MV 2469 & Original isolate & & & Cockles \\
\hline 33 & MV 2476 & Vibrio sp. & & (5) & River water \\
\hline 34 & MV 2477 & Vibrio sp. & & (5) & River water \\
\hline 35 & MV 2478 & Vibrio sp. & & (5) & River water \\
\hline 36 & MV 2480 & Vibrio sp. & & (5) & River water \\
\hline 37 & MV 2481 & Vibrio sp. & & (5) & River water \\
\hline 38 & MV 2484 & Original isolate & & & Cockles \\
\hline 39 & MV 2488 & Original isolate & & & Prawns \\
\hline 40 & MV 400 & $V$. cholerae classical & & (7) & Feces, human \\
\hline 41 & MV 1208 & $V$. cholerae classical & NCTC 7254 & (1) & Feces, human \\
\hline 42 & MV 1330 & $V$. cholerae eltor & NCTC 10954 & (1) & Feces, human \\
\hline 43 & MV 1214 & V. cholerae (NCV) & & (8) & Feces, human \\
\hline 44 & MV 1401 & V. cholerae (NCV) & & & Feces, human \\
\hline 45 & MV 1479 & V. cholerae (NCV) & & & Prawns \\
\hline 46 & MV 463 & $V$. alginolyticus & & (9) & Seawater \\
\hline 47 & MV 1251 & V. alginolyticus & & & Oysters \\
\hline 48 & MV 2204 & V. alginolyticus & ATCC 17749 & (3) & $\begin{array}{l}\text { Spoiled horse } \\
\text { mackerel }\end{array}$ \\
\hline 49 & MV 515 & V. parahaemolyticus & & & Feces, human \\
\hline 50 & MV 1225 & V. parahaemolyticus & & & Feces, human \\
\hline 51 & MV 1424 & V.parahaemolyticus & ATCC 17802 & (3) & Feces, human \\
\hline 52 & MV 1490 & $V$. anguillarum & NCMB 6 & $(10)$ & Diseased cod \\
\hline 53 & MV 2045 & V. anguillarum & & & Oyster \\
\hline 54 & MV 2176 & $V$. anguillarum & & & Clam \\
\hline 55 & AM 2206 & $V$. anguillarum & & & Clam \\
\hline 56 & MV 2600 & $V$. anguillarum & & (5) & Mussel \\
\hline 62 & MV 1488 & $V$. fischeri & NCMB 1143 & (10) & Pacific cod \\
\hline 63 & MV 1493 & V. fischeri & & & Prawns \\
\hline
\end{tabular}


TABLE $1-($ Continued $)$

\begin{tabular}{|c|c|c|c|c|c|}
\hline \multirow{2}{*}{ Computer no. } & \multirow{2}{*}{ Laboratory no. } & \multicolumn{2}{|c|}{ Received as: } & \multirow{2}{*}{ Source $^{a}$} & \multirow{2}{*}{ Habitat } \\
\hline & & Name & Strain & & \\
\hline \multicolumn{6}{|l|}{ Aeromonas species } \\
\hline 64 & MV 1121 & A. hydrophila & NCTC 8049 & (1) & Milk \\
\hline 65 & MV 1122 & A. hydrophila & NCTC 7810 & (1) & Frog "red leg" \\
\hline 66 & MV 2071 & Original isolate & & & Feces, human \\
\hline 67 & MV 2169 & Original isolate & & & Feces, human \\
\hline 68 & MV 2415 & & & (5) & River water \\
\hline 69 & MV 1133 & Original isolate & & & Feces, human \\
\hline 70 & MV 1140 & Original isolate & & & Prawns \\
\hline 71 & MV 1931 & Original isolate & & & River water \\
\hline 72 & MV 1933 & Original isolate & & & River water \\
\hline 73 & MV 2224 & & & (4) & Sewage \\
\hline 74 & MV 2374 & & & (4) & Sewage \\
\hline \multicolumn{6}{|c|}{$\begin{array}{c}\text { Beneckea species and } \\
\text { others }\end{array}$} \\
\hline 57 & MV 2386 & & & (5) & River water \\
\hline 58 & MV 2387 & & & (5) & River water \\
\hline 59 & MV 2389 & & & (5) & River water \\
\hline 60 & MV 2391 & & & (5) & River water \\
\hline 61 & MV 2418 & & & (5) & River water \\
\hline 75 & MV 2189 & B. campbellii & ATCC 25920 & (3) & Seawater \\
\hline 76 & MV 2190 & B. natriegens & ATCC 14048 & (3) & $\underset{\text { mud }}{\text { Salt marsh }}$ \\
\hline 77 & MV 2191 & B. neptuna & ATCC 25919 & (3) & Seawater \\
\hline 78 & MV 2192 & B. nereida & ATCC 25917 & (3) & Seawater \\
\hline 79 & MV 2193 & B. nigrapulchrituda & ATCC 27043 & (3) & Seawater \\
\hline 80 & MV 2194 & B. pelagia & ATCC 25916 & (3) & Seawater \\
\hline 81 & MV 2195 & $\begin{array}{l}\text { Group B2 of Bau- } \\
\text { mann et al. (1) }\end{array}$ & ATCC 25915 & (3) & Seawater \\
\hline 82 & & $\begin{array}{c}\text { Photobacterium } \\
\text { phosphoreum }\end{array}$ & NCMB 844 & (10) & \\
\hline 83 & & Escherichia coli & NCTC 10418 & (1) & \\
\hline
\end{tabular}

a (1) NCTC, National Collection of Type Cultures, Central Public Health Laboratory, London, England. (2) P, Institut Pasteur, Paris, France. (3) ATCC, American Type Culture Collection, Rockville, Md. (4) V. Muić, Institute of Public Health of SR Croatia, Zagreb, Yugoslavia. (5) J. McCoy, Public Health Laboratory, Hull Royal Infirmary, Hull, England. (6) R. Hugh, Department of Microbiology, George Washington University School of Medicine, Washington D.C. (7) Cholera Research Laboratory, Dacca, Bangladesh. (8) School of Public Health and Tropical Medicine, Sydney, Australia. (9) E. Aldova, Institute of Epidemiology and Microbiology, Prague, Czechoslovakia. (10) National Collection of Marine Bacteria, Torry Research Station, Aberdeen, Scotland.

media were inoculated as for the salt tolerance test. Growth was recorded after incubation for 14 days at $4^{\circ} \mathrm{C}, 2$ days at $15^{\circ} \mathrm{C}$, and 1 day at 30,37 , and $42^{\circ} \mathrm{C}$.

Swarming. Marine agar was prepared according to the manufacturer's instructions, and the sterile molten agar was cooled in a water bath at $45^{\circ} \mathrm{C}$ for 1 $h$ before the plates were poured. After the plates had set, they were not dried, but gutters were cut in them to divide the agar into quadrants. Each quadrant was inoculated with a 10-mm streak of one of the test organisms. Plates were examined for swarming after overnight incubation. A known positive control organism was included with each batch of strains tested.

Luminescence. Strains were inoculated onto plates of nutrient agar containing (per liter): nutrient broth dried base (Oxoid), $25 \mathrm{~g} ; \mathrm{NaCl}, 17.5 \mathrm{~g} ; \mathrm{KCl}, 1.0$ g; $\mathrm{MgCl}_{2} \cdot 6 \mathrm{H}_{2} \mathrm{O}, 4.0 \mathrm{~g}$; Davis agar, $15.0 \mathrm{~g}$. After overnight incubation at $20^{\circ} \mathrm{C}$, luminescence was recorded after the plates were observed for $5 \mathrm{~min}$ in the dark. Antibiotic susceptibilities. Susceptibility to 2,4diamino-6,7-diisopropyl-pteridine phosphate (0/129) was determined on heart infusion agar (Difco). Susceptibilities to other antibiotics were determined on Sensitest agar (Oxoid). Strains 53, 62, 78, 79, 81, and 82 would not grow on this medium and were tested on marine agar. The antibiotics and the concentrations used are shown in Table 2. For novobiocin, 0/129, and methylene blue, the susceptibilities were read against $V$. cholerae 41 (NCTC 1208) as a control. For the remaining antibiotics, Escherichia coli 83 (NCTC 10418) was used.

General physiological and biochemical properties. Unless otherwise stated, tests were read during an incubation period of 5 days.

The mode of glucose metabolism was examined by the method of Hugh and Leifson (10), but the $\mathrm{NaCl}$ 
concentration of the medium was increased to $3 \%$ (wt/vol).

Strains were tested for: growth after $24 \mathrm{~h}$ on TCBS, cystine-lactose electrolyte-deficient medium, and MacConkey agar (all Oxoid); hemolysis of sheep erythrocytes, as determined by the method of Sakazaki et al. (21); agglutination of chicken erythrocytes by the method of Finkelstein and Mukerjee (7); chitinase production on MA overlaid with MA containing $1 \%$ (wt/vol) colloidal chitin; alginase on MA overlaid with MA containing $2 \%$ (wt/vol) sodium alginate; lecithinase on Oxoid blood agar base CM55 containing $1 \%$ (wt/vol) $\mathrm{NaCl}$ and $10 \%$ (wt/vol) egg yolk emulsion (Oxoid); arginine dihydrolase by the method of Thornley (25); and the reduction of trimethylamine oxide to trimethylamine by the method of Laycock and Regier (15), except that $20 \%$ (wt/vol) $\mathrm{KOH}$ was used instead of $\mathrm{K}_{2} \mathrm{CO}_{3}$ (18).

The following tests were performed as described by Cowan (5). When alternatives are listed, the one chosen is shown in parentheses. The sodium chloride concentration in all the media was raised to $1 \%$ (wt/vol). The tests were: indole production after $24 \mathrm{~h}$ (method 2); Kovács (14) oxidase; catalase (method 1); reduction of nitrate to nitrite, but using reagents $A$ and B of Crosby (6); acid and gas production from carbohydrates in peptone water containing Andrade indicator; $O$-nitrophenyl- $\beta$-D-galactopyranoside; Voges-Proskauer after 1 day (method 1); methyl red after 2 days of incubation; gluconate oxidation (method 2); decarboxylation of arginine, lysine, and ornithine (method 1), using Difco decarboxylase base; urease (method 1); hydrogen sulfide production (method 1); deoxyribonuclease on Difco deoxyribonuclease test agar; gelatinase $(\operatorname{method} 3)$; Tween 80 hydrolysis (method 1); phosphatase (method 1), substituting MA for nutrient agar; citrate utilization (method 2); malonate utilization (method 1); phenylalanine deamination (method 1); starch hydrolysis (method 1), substituting MA for nutrient agar.

Utilization of carbon compound. Strains were screened for their ability to utilize 49 different compounds as the sole organic source of carbon, using the basal medium of Baumann et al. (1). All of the strains studied were capable of growth on basal medium containing $0.1 \%$ (wt/vol) vitamin-free Casamino Acids (Difco) and were therefore considered to have no vitamin requirements. If sufficiently soluble, the substrates were prepared as concentrated solutions, which were sterilized by passage through a $0.2-\mu \mathrm{m}$-pore membrane filter (Sartorius) and added to the medium immediately before plates were poured. Otherwise, compounds were dissolved in the medium and autoclaved at $115^{\circ} \mathrm{C}$ for $10 \mathrm{~min}$. In all cases the final concentration of the substrate in the medium was $0.1^{\%}(\mathrm{wt} / \mathrm{vol})$. Plates of medium were inoculated by means of a mechanical multipoint inoculator. Strains were grown in $T_{1} N_{2}$, and the inoculum was prepared by diluting the culture with fresh $T_{1} N_{2}$ such that the final inoculum on the plate contained approximately $10^{3}$ organisms in a spot of $2-$ to $3-\mathrm{mm}$ diameter. Up to 24 strains could be inoculated onto one $90-\mathrm{mm}$ diameter petri dish. Strains that swarmed were isolated from other strains by gutters in the agar, and only six were then tested on one plate. Growth was recorded for 7 days. A positive (basal medium plus $0.1 \%$ vitamin-free Casamino Acids) control and a negative (basal medium only) control were put up for every strain.

Base composition of DNA. The deoxyribonucleic acid (DNA) base compositions of selected strains were determined by thermal denaturation (17) after extraction and purification by the method of Marmur (16).

Numerical analysis of results. Results from all the tests shown in Table 2, except mode of flagellation, were used in a computer-assisted taxonomic analysis. The results were regarded as two-state characters with the exception of temperature range and salt tolerance. Temperature range was coded as two multistate quantitative characters, i.e., the minimum and maximum temperatures at which the strains grew. Growth in the absence of sodium chloride was considered as a single two-state character, and growth at the remaining salt concentrations was coded as two multistate quantitative characters, i.e., the minimum and maximum salt concentrations in which the strains grew. If a strain grew in the absence of sodium chloride, the character "salt minimum" was coded as "no comparison," as was the character "production of gas from glycerol" if the strain did not produce acid from glycerol, and as was the character "acid from lactose" if the strain was negative in the ONPG test. Similarities were calculated by using the general similarity coefficient of Gower (negative matches were counted for all two-state characters), and dendrograms were constructed by single-linkage and weighted pair-group centroid (median) clustering methods (24).

(The full list of code characters and strain data have been deposited with the World Data Center for Microorganisms, Department of Microbiology, University of Queensland, St. Lucia Brisbane 4067, Australia, from which it can be obtained.)

\section{RESULTS AND DISCUSSION}

The characteristics of the strains are shown in Table 2. The two methods of cluster analysis, with few exceptions, gave identical groupings (Fig. 1 and 2). We shall therefore confine our discussion to the dendrogram constructed from the single-linkage cluster analysis (Fig. 1). The clustering is very much as we would have expected from our experience in identifying members of the Vibrionaceae and from the work of others $(1,4,20)$. Seven major phenons, described below, were formed in the analysis.

V. metschnikovii. The naming of the phenon V. metschnikovii and its taxonomic position are discussed below. It is formed at the $89 \%$ level of similarity (S), contains only oxidasenegative, vibrio-like organisms, and includes all such organisms studied with one exception. Within this phenon there are two subphenons. The smaller one formed at $92 \% \mathrm{~S}$ contains all of the $V$. metschnikovii strains, no. 29 received as $V$. tyrogenes and no. 26 received as "Vibrio sp. (cholera gp.)." The second subphenon formed at $91 \% \mathrm{~S}$ includes all of the wild strains 
TABLE 2. Characteristics of V. metschnikovii and the other phenons ${ }^{a}$

\begin{tabular}{|c|c|c|c|c|c|c|c|c|c|c|c|c|c|c|c|c|}
\hline Determination & 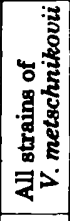 & 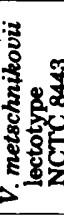 & 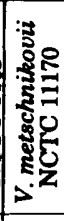 & 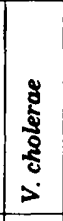 & 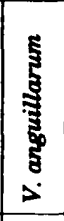 & $\begin{array}{c}0 \\
0 \\
3 \\
5 \\
5 \\
5\end{array}$ & 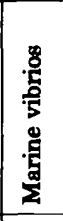 & 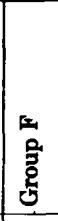 & 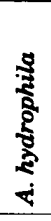 & 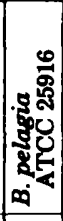 & 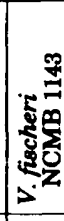 & 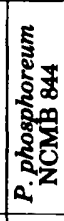 & 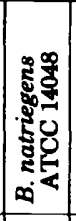 & 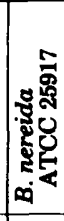 & 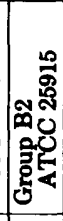 & 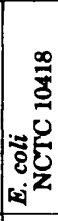 \\
\hline No. of strains & 39 & 1 & 1 & 6 & 5 & 6 & 10 & 6 & 4 & 1 & 1 & 1 & 1 & 1 & 1 & 1 \\
\hline owth on: & 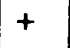 & + & + & + & + & V & + & + & + & + & + & + & + & + & + & + \\
\hline TCBS & +- & - & + & + & + & +- & + & + & $\mathbf{v}$ & + & - & - & + & - & - & - \\
\hline CLE & + & + & + & t & - & + & - & + & + & - & - & - & - & - & - & + \\
\hline MacConkey & +- & - & + & + & -+ & + & - & + & + & - & - & - & - & - & - & + \\
\hline Swarmi & - & - & - & - & - & - & -+ & - & - & - & - & - & - & - & - & - \\
\hline Lumin & - & - & - & - & - & - & - & - & - & - & + & + & - & - & - & - \\
\hline Growth in $\% \mathrm{NaCl}$ : & - & - & - & + & - & + & - & - & + & - & - & & - & & & \\
\hline $\begin{array}{l}0 \\
0.5\end{array}$ & $\bar{t}$ & $\overline{+}$ & + & $\begin{array}{l}+ \\
+\end{array}$ & $\bar{t}$ & $\begin{array}{l}+ \\
+\end{array}$ & + & + & $\begin{array}{l}+ \\
+\end{array}$ & - & $\overline{-}$ & + & $\overline{+}$ & $\overline{-}$ & $\begin{array}{l}- \\
-\end{array}$ & $\begin{array}{l}+ \\
+\end{array}$ \\
\hline 2 & + & + & + & + & + & + & + & + & + & + & + & + & + & + & + & + \\
\hline 3 & + & + & + & + & + & + & + & + & + & + & + & + & + & + & + & + \\
\hline 4 & + & + & + & +- & + & + & + & + & V & + & + & + & + & + & + & + \\
\hline 5 & + & + & + & V & + & -+ & + & + & - & + & - & + & + & + & + & + \\
\hline 6 & + & + & + & V & + & - & +- & + & - & + & - & - & + & + & - & + \\
\hline 7 & + & + & + & -+ & + & - & +- & + & - & - & - & - & + & + & - & + \\
\hline 8 & V & - & + & - & -+ & - & V & + & - & - & - & - & + & + & - & - \\
\hline 10 & - & - & - & - & - & - & V & -+ & - & - & - & - & - & + & - & - \\
\hline Grow & & & & & & & & & & & & & & & & \\
\hline $1:$ & +- & + & + & -+ & + & +- & v & + & V & - & + & + & + & + & - & - \\
\hline & + & + & + & + & + & + & + & + & + & + & - & - & + & + & + & + \\
\hline 3 & + & + & + & + & +- & + & +- & + & + & - & - & - & + & + & - & + \\
\hline $42^{\circ} \mathrm{C}$ & V & - & + & + & - & V & V & $\mathrm{V}$ & V & - & - & - & - & - & - & - \\
\hline Hemolysis & + & + & + & V & +- & V & - & - & V & - & NC & $\mathrm{NC}$ & - & + & + & - \\
\hline Catale & + & + & + & + & + & + & + & + & + & + & + & + & - & - & - & + \\
\hline O & - & - & - & + & + & + & + & + & + & + & + & - & + & + & - & - \\
\hline No & - & - & - & + & + & + & + & + & + & + & + & + & + & + & - & + \\
\hline TMA & - & - & - & +- & + & + & + & + & + & + & + & + & + & + & + & + \\
\hline $\mathbf{M}$ & +- & - & + & + & V & +- & + & + & V & + & - & + & + & + & + & + \\
\hline $\mathrm{VI}$ & + & + & + & $\mathrm{V}$ & +- & + & -+ & + & + & - & - & - & - & - & + & + \\
\hline Indol & V & + & - & + & +- & + & + & +- & + & - & - & - & - & + & - & + \\
\hline ONPG & +- & + & + & + & +- & + & V & + & + & + & + & - & - & NC & - & + \\
\hline Gas & & & & & & & & & & & & & & & & \\
\hline & - & - & - & - & - & V & - & +- & + & - & - & + & - & - & - & + \\
\hline G & - & - & - & - & - & - & - & -+ & + & - & - & - & - & - & - & - \\
\hline Ac & & & & & & & & & & & & & & & & \\
\hline Ar & - & - & - & - & - & V & $\mathrm{v}$ & + & $\mathrm{V}$ & - & - & - & - & - & - & + \\
\hline & - & - & - & - & +- & +- & + & -+ & - & - & + & - & + & - & - & - \\
\hline $\mathrm{D}$ & - & - & - & - & - & - & - & -+ & - & - & - & - & - & - & - & + \\
\hline & + & + & + & - & - & V & v & +- & + & - & - & - & - & - & - & - \\
\hline Inc & v & + & - & - & - & - & - & - & - & - & - & - & - & - & - & - \\
\hline & $\mathrm{v}$ & + & + & - & - & V & - & - & V & - & - & 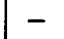 & - & - & - & + \\
\hline $10 \%$ Lactose & +- & + & + & V & - & V & - & - & V & - & - & - & - & - & + & + \\
\hline & 1 & + & + & + & +- & + & + & + & + & + & + & + & + & + & + & + \\
\hline $\mathbf{M} \varepsilon$ & + & + & + & + & + & + & + & + & + & + & - & - & + & - & - & + \\
\hline & +- & + & + & +- & +- & V & + & + & + & - & - & + & - & - & + & + \\
\hline Raffinose & 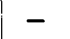 & - & - & - & - & - & - & - & - & - & - & - & + & - & - & + \\
\hline & 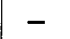 & 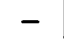 & - & - & - & - & - & -+ & - & - & - & - & + & - & - & + \\
\hline Salic & & 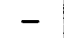 & - & - & - & -+ & - & - & - & - & - & - & + & - & - & - \\
\hline & -7 & - & - & - & v & - & - & - & - & - & - & - & - & - & - & + \\
\hline Starch & + & + & + & +- & + & + & + & + & + & + & + & - & + & + & - & - \\
\hline & 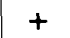 & + & + & +- & + & +- & v & + & + & + & + & - & + & + & + & + \\
\hline Trehalose & + & + & + & + & + & + & + & + & + & + & + & - & + & + & - & + \\
\hline & & & - & - & - & - & - & - & - & - & - & - & - & - & - & + \\
\hline Gluconate oxidation & - & - & -1 & - & - & - & - & - & + & - & - & - & - & - & - & - \\
\hline
\end{tabular}


TABLE 2-(Continued)

\begin{tabular}{|c|c|c|c|c|c|c|c|c|c|c|c|c|c|c|c|c|}
\hline Determination & 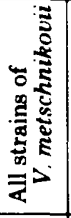 & 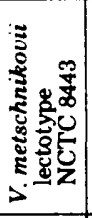 & 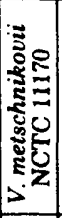 & 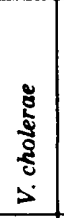 & 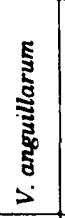 & $\begin{array}{l}0 \\
0 \\
\text { ํํํ } \\
0\end{array}$ & 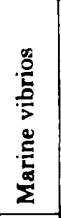 & $\begin{array}{l}0 \\
0 \\
0 \\
0 \\
0\end{array}$ & 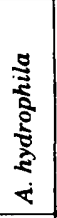 & 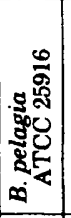 & 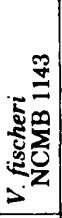 & 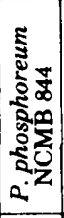 & 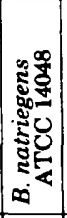 & 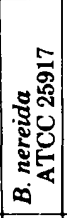 & 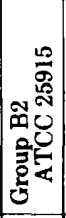 & 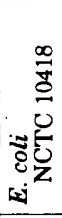 \\
\hline $\begin{array}{l}\text { Thornley's arginine } \\
\text { Moeller's }\end{array}$ & +- & + & + & - & + & + & - & + & + & - & - & + & - & + & - & - \\
\hline Arginine & +- & + & + & - & +- & + & - & + & + & - & - & + & - & + & + & - \\
\hline Lysine & +- & + & + & + & -+ & -+ & + & - & V & - & + & + & - & - & - & + \\
\hline Ornithine & - & - & - & + & - & - & V & - & - & - & - & - & - & - & - & + \\
\hline Amylase & + & + & + & +- & + & +- & + & V & + & - & - & - & - & - & - & - \\
\hline Alginase & - & - & - & - & - & - & - & - & - & + & - & - & - & - & - & - \\
\hline Chitinase & + & + & + & +- & +- & + & + & + & + & + & - & + & - & + & + & - \\
\hline Deoxyribonuclease & + & + & + & + & + & + & + & + & + & + & + & + & + & + & + & - \\
\hline Gelatinase & + & + & + & + & + & + & + & + & + & - & - & NC & + & + & - & - \\
\hline Lecithinase & + & + & + & +- & V & -+ & + & + & $\mathrm{V}$ & - & + & + & - & - & + & - \\
\hline Tween 80 & + & + & + & + & +- & + & + & + & + & + & + & - & + & - & - & - \\
\hline Phosphatase & V & + & - & + & + & + & + & + & + & + & + & - & + & + & + & - \\
\hline Urease & - & - & - & - & - & -+ & -+ & - & - & - & - & - & - & - & - & - \\
\hline Malonate & - & - & - & - & V & - & - & V & - & - & $\mathrm{NC}$ & - & - & - & - & - \\
\hline PPA & - & - & - & - & - & - & - & - & V & - & NC & - & - & - & - & - \\
\hline Simmons' citrate & V & - & + & + & - & + & - & $\mathrm{V}$ & V & - & - & - & - & - & - & - \\
\hline CCA & -+ & - & - & V & V & V & - & - & V & - & + & - & - & - & - & + \\
\hline Susceptibility to: & & & & & & & & & & & & & & & & \\
\hline Ampicillin, $10 \mu \mathrm{g}$ & V & + & - & + & V & V & V & + & - & + & + & + & + & + & + & + \\
\hline Carbenicillin, $100 \mu g$ & +- & + & - & + & V & V & V & + & - & + & + & + & + & + & + & + \\
\hline Cephaloridine, $15 \mu g$ & + & + & + & + & -+ & V & + & + & V & + & + & + & + & + & - & + \\
\hline Kanamycin, $30 \mu \mathrm{g}$ & + & + & + & + & + & + & + & + & + & + & - & + & + & + & + & + \\
\hline Methylene blue, $100 \mu g$ & + & + & + & + & V & - & + & - & $\mathrm{V}$ & + & + & + & + & + & + & - \\
\hline Novobiocin, $5 \mu \mathrm{g}$ & + & + & + & + & +- & -+ & +- & - & $\mathrm{V}$ & + & + & + & - & + & + & - \\
\hline B, 50 IU & + & + & + & V & + & + & +- & + & + & + & NC & + & + & + & + & + \\
\hline Streptor & + & + & + & + & + & + & + & + & + & + & + & + & + & + & + & + \\
\hline Sulfafure & + & + & + & V & + & +- & + & + & V & + & + & + & + & + & + & + \\
\hline Tetracycline, $10 \mu 8$ & + & + & + & + & +- & +- & + & + & + & + & + & + & + & + & + & + \\
\hline Trimethoprim, $1.25 \mu \mathrm{g}$ & + & + & + & + & + & -+ & +- & + & - & + & + & + & + & + & - & + \\
\hline $0 / 129,100 \mu \mathrm{g}$ & + & + & + & + & + & - & + & V & - & + & + & + & + & + & + & - \\
\hline Growth on: & & & & & & & & & & & & & & & & \\
\hline Alanine & + & + & + & - & V & - & +- & + & - & - & - & - & + & + & - & + \\
\hline Arginine & -+ & + & - & - & - & - & + & + & - & + & - & - & + & + & - & - \\
\hline Asparagine & + & - & + & +-1 & + & -+ & + & + & V & + & - & - & + & + & + & - \\
\hline Aspartate & - & - & - & -+ & -+ & - & V & + & - & + & - & - & - & + & + & - \\
\hline Glutamate & +- & + & + & V & + & V & + & + & - & + & + & + & + & + & + & - \\
\hline Glutamine & +- & - & + & V & + & V & + & + & - & + & - & - & + & + & + & - \\
\hline Glycine & - & - & - & - & - & - & + & V & V & + & - & - & + & + & - & - \\
\hline Histidine & -+ & + & - & +- & NC & V & + & NC & NC & $\mathrm{NC}$ & - & - & NC & NC & NC & - \\
\hline Leucine & - & - & - & - & - & - & V & - & - & - & - & - & + & + & - & - \\
\hline Lysine & - & - & - & - & - & - & - & - & - & - & - & - & - & + & - & - \\
\hline Proline & + & + & + & +- & + & V & + & + & V & + & - & - & + & + & + & + \\
\hline Putrescine & - & - & - & - & -+ & - & V & + & - & + & - & - & + & + & - & + \\
\hline Serine & V & - & + & V & + & V & + & + & - & + & - & - & + & + & - & - \\
\hline Threonine & + & + & + & - & + & - & + & + & V & + & - & - & + & + & + & - \\
\hline Arabinose & - & - & - & - & - & V & V & + & V & - & - & - & + & - & - & + \\
\hline Cellobiose & - & - & - & - & +- & +- & V & - & - & - & - & - & + & - & - & - \\
\hline Galactose & - & - & - & - & -+ & - & V & + & - & - & - & + & - & - & + & - \\
\hline Gluconate & + & + & + & + & +- & + & +- & + & - & + & - & - & + & + & - & + \\
\hline Glucose & + & + & + & + & + & + & + & + & - & + & - & + & + & + & + & + \\
\hline Glucuronate & - & - & - & -+ & - & - & $\mathbf{V}$ & - & - & + & - & - & - & - & - & - \\
\hline Glycerol & +- & + & + & +- & + & + & + & + & V & + & - & + & + & + & + & + \\
\hline Inositol & $\mathbf{V}$ & + & - & - & - & - & - & - & V & - & - & - & - & - & - & - \\
\hline Lactose & V & - & + & - & -+ & V & - & - & V & + & - & - & - & - & - & + \\
\hline Salicin & - & - & - & - & - & V & -+ & - & - & + & - & - & + & + & - & - \\
\hline
\end{tabular}




\begin{tabular}{|c|c|c|c|c|c|c|c|c|c|c|c|c|c|c|c|c|}
\hline Determination & 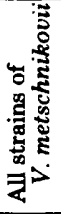 & 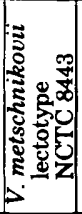 & 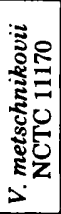 & 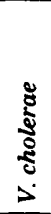 & 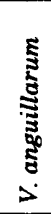 & $\mid \begin{array}{l}0 \\
0 \\
3 \\
0 \\
0 \\
0\end{array}$ & 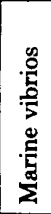 & $\begin{array}{l}5 \\
\frac{2}{3} \\
\vdots \\
5 \\
5\end{array}$ & 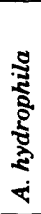 & 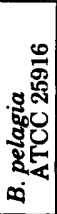 & 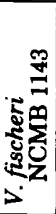 & 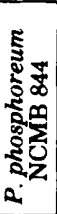 & 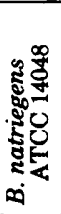 & 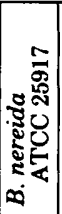 & 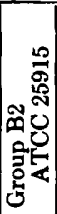 & 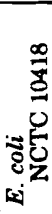 \\
\hline Sorbitol & -+ & - & - & - & V & - & - & - & - & + & - & - & - & - & + & - \\
\hline Sucrose & + & + & + & +- & + & +- & V & + & - & + & - & - & + & + & + & + \\
\hline Xylose & - & - & - & - & - & - & - & - & - & - & - & - & - & - & + & + \\
\hline Acetate & +- & - & + & + & +- & -+ & + & + & - & + & - & - & + & + & + & + \\
\hline Butyrate & - & - & - & - & - & - & V & - & - & - & - & - & + & + & - & - \\
\hline Decanoate & +- & - & + & - & - & $\mathrm{V}$ & +- & + & - & - & - & - & + & + & - & - \\
\hline Formate & - & - & - & - & - & - & - & - & - & - & - & - & - & - & - & - \\
\hline$p$-OH benzoate & - & - & - & - & - & - & - & V & - & - & - & - & + & - & - & - \\
\hline 3-OH butyrate & - & - & - & - & - & - & - & V & - & + & - & - & + & + & - & - \\
\hline$\alpha$-Ketoglutarate & - & - & - & +- & $\mathrm{V}$ & - & + & + & V & + & - & - & + & + & + & + \\
\hline Pyruvate & +- & + & + & $\mathbf{V}$ & - & - & -+ & V & - & - & - & - & - & + & - & - \\
\hline Succinate & +- & - & + & +- & + & + & V & + & - & + & - & - & + & + & + & - \\
\hline Ethanol & - & - & - & - & - & - & V & + & - & - & - & - & - & + & - & - \\
\hline Propanol & - & - & - & - & - & - & -+ & V & - & - & - & - & - & + & - & - \\
\hline Flagella & $\mathbf{m}$ & $\mathbf{m}$ & $\mathbf{m}$ & $m$ & $\mathbf{m}$ & m & $\mathbf{m}$ & $\mathbf{m}$ & $\mathbf{m}$ & $\mathbf{m}$ & $\mathbf{m}$ & $\mathbf{m}$ & $\mathbf{m}$ & $\mathrm{m}$ & $\mathbf{m}$ & $\mathbf{p}$ \\
\hline
\end{tabular}

${ }^{a}$ All strains were positive for: glucose fermentation; growth in $1 \% \mathrm{NaCl}$; susceptibility to nalidixic acid, septrin, nitrofurantoin, chloramphenicol, and gentamicin. All strains were negative for: Gram reaction: adonitol (peptone-water-sugar); $\mathrm{H}_{2} \mathrm{~S}$; growth at $4^{\circ} \mathrm{C}$; growth on cysteine, isoleucine, methionine, phenylalanine, tryptophan, tyrosine, valine, DL-2-hydroxybutyrate, tartrate, butanol, and erythrose. Symbols/abbreviations: + , reaction positive for at least $90 \%$ of strains; -, reaction negative for at least $90 \%$ of strains; $V$, variable reaction for group; +- or -+, first reaction present in 80 to $89 \%$ of the strains; $\mathrm{m}$, monotrichous polar flagella; $p$, peritrichous flagella; CLED, cystine-lactose electrolyte-deficient medium; TMAO, trimethylamine oxide; MR, methyl red; VP, Voges-Proskauer; ONPG, $o$-nitrophenyl- $\beta$-D-galactopyranoside; PPA, phenyl pyruvic acid test; CCA, chick cell agglutination.

${ }^{b}$ Included in total for phenon.

and the $V$. proteus strains, the latter forming a single cluster at $94 \% \mathrm{~S}$.

Number 81 (ATCC 25915), which represents group B2 of Baumann et al. (1), was the only oxidase-negative, vibrio-like strain in our study that did not cluster in the $V$. metschnikovii phenon. It is clearly not closely related to any of the organisms in the study since it is the last but one strain to cluster.

V. cholerae. The phenon $V$. cholerae contains all the strains of the classical and eltor biotypes of $V$. cholerae, including the type strain, and all the so-called noncholera vibrios and no others. It is now recognized that the noncholera vibrios, also called non-agglutinable vibrios by some workers, should be considered as different serotypes of the species $V$. cholerae (13). This phenon therefore corresponds to $V$. cholerae.

V. anguillarum. The phenon $V$. anguillarum, formed at $84 \% \mathrm{~S}$, consists of the four wild strains we had previously identified as $V$. anguillarum and no. 52 (NCMB 6), one of the strains recommended as a working type by Shewan and Véron (23). Numbers 54 and 53, which were the last to join the $V$. anguillarum phenon by single linkage, are separated from it by me- dian sorting (Fig. 1 and 2). This suggests that the phenon may be slightly heterogenous, but it corresponds to $V$. anguillarum as described by Shewan and Véron (23).

Group D. The phenon group D contains six strains that we would identify as Aeromonas, but no type strains are included.

Marine vibrios. The rather heterogenous group marine vibrios contains the type strains and all the other strains of $V$. alginolyticus and $V$.parahaemolyticus, along with the type strains of Beneckea neptuna (no. 77), B. campbellii (no. 75), and B. nigrapulchrituda (no. 79). The remaining strain in this group is no. 63. It is luminescent and closely matches the description of $V$. fischeri (23), except that it grows at $37^{\circ} \mathrm{C}$ and possesses ornithine decarboxylase. The particular strain of $V$. fischeri used in the study (no. 62, NCMB 1143) was rather unreactive and differs from the published description in being negative for methyl red, gelatinase production, and acid production from mannose. Number 62 did not fall into any distinct phenon in our study.

The marine vibrio phenon has no clear subcluster within it corresponding to the species $V$. parahaemolyticus or V. alginolyticus. Probably, with a much larger number of strains, subclus- 


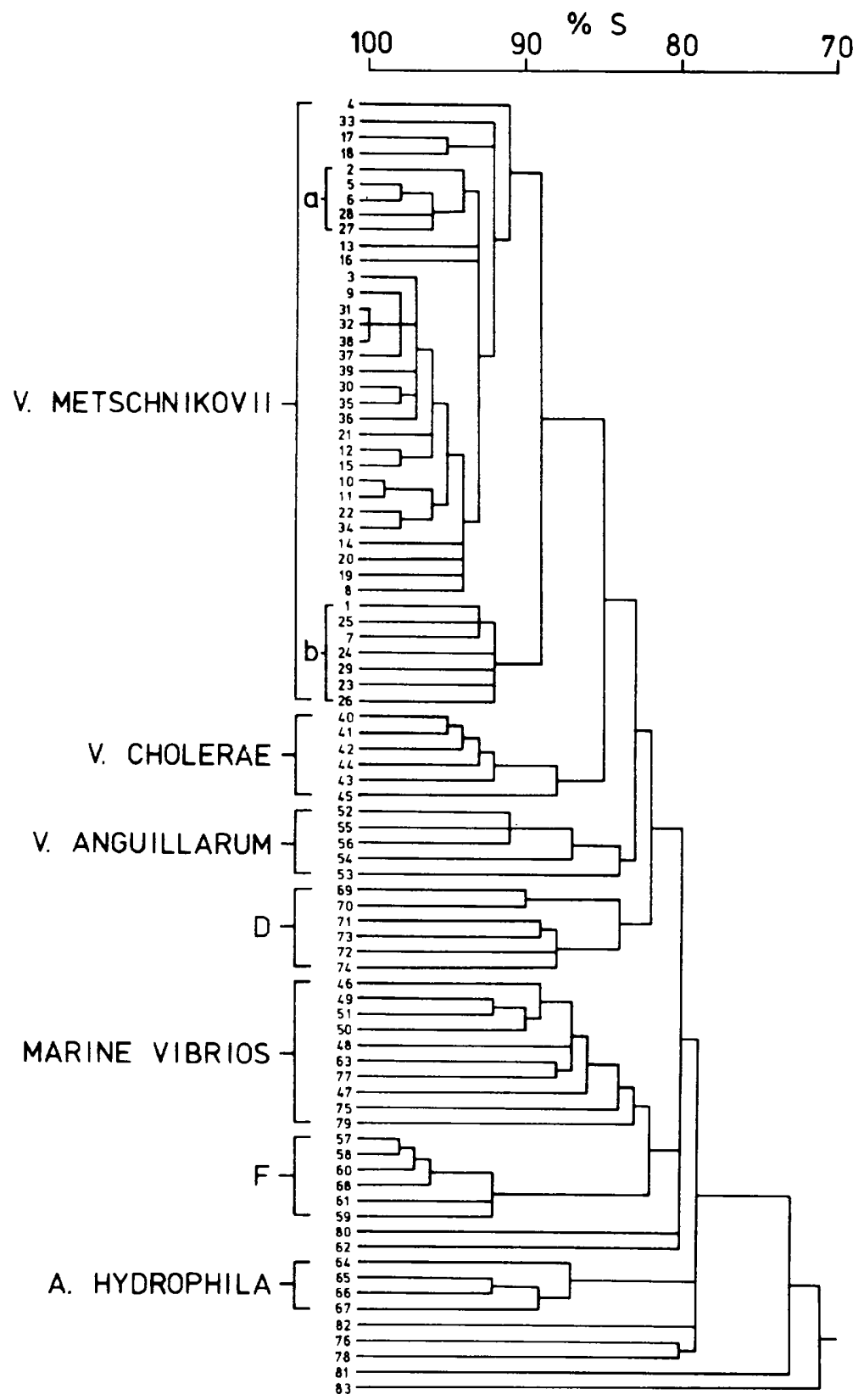

FIG. 1. Dendrogram constructed by single-linkage cluster analysis. (a) Culture collection strains of V. proteus; (b) culture collection strains of $V$. metschnikovii.

ters would have been formed within this phenon corresponding to the species represented in it. Nevertheless over the past 2 years, in attempting to identify many hundreds of vibrio isolates from the marine environment, we have found very many strains with characteristics between $V$. alginolyticus, $V$. parahaemolyticus, B. neptuna, $B$. campbellii, and $V$. fischeri. This marine vibrio phenon is therefore much as we would have expected.

Group F. Group F is a very interesting group, which we had not suspected existed until it was revealed by this study and another one running concurrently in our laboratory on the antibiotic susceptibilities of vibrios. It includes six strains of which five produce gas from glucose, and all 


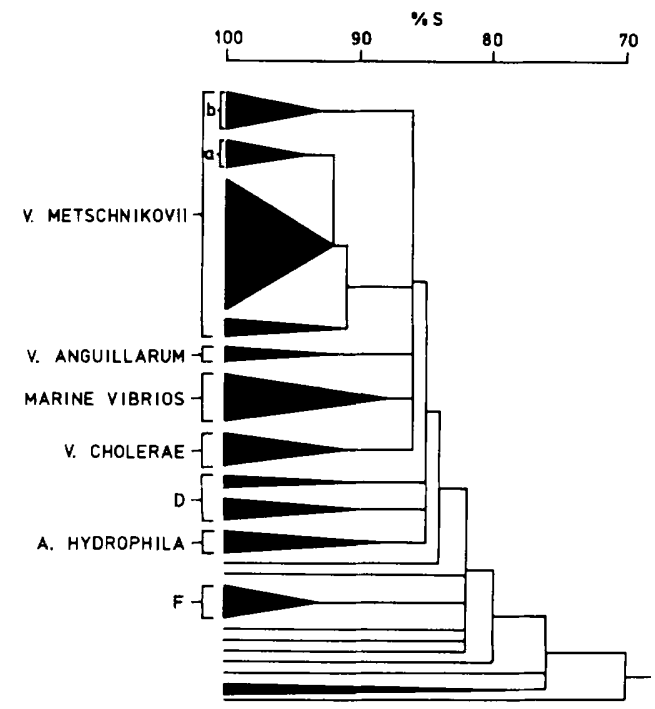

FIG. 2. Dendrogram constructed by weighted pairgroup centroid (median) cluster analysis. (a) Culture collection strains of $V$. proteus; (b) culture collection strains of $V$. metschnikovii.

six had previously been identified as Aeromonas spp. However, unlike other aeromonads, they are unable to grow in the absence of $\mathrm{NaCl}$, they can grow on a much wider range of compounds as the sole organic source of carbon, and they show some degree of susceptibility to $0 / 129$. In this study they are recorded as having variable susceptibility to $0 / 129$ when tested with disks containing $100 \mu \mathrm{g}$ of the drug. If tested with disks containing $150 \mu \mathrm{g}$, all of these strains are susceptible, whereas Aeromonas strains remain resistant.

We now realize that this group is extremely widely distributed in the marine and estuarine environment around Britain. We have also received similar strains from patients with diarrhea whose sources of infection were in Bangladesh, Bahrein, and Jordan. Two of the strains from this study and one from a patient whose illness was contracted in Bahrein have had their DNA base composition examined. The guanineplus-cytosine $(\mathrm{G}+\mathrm{C})$ content of the DNA of these strains was in the range 51.5 to $52.2 \mathrm{~mol} \%$ (Table 3). This is too low for the genus Aeromonas (57 to $63 \mathrm{~mol} \%$ ) and perhaps too high for the genus Vibrio, which has 40 to $50 \mathrm{~mol} \%$ $\mathrm{G}+\mathrm{C}(22,23)$. It seems probable that they constitute a new species if not a new genus. However, at the moment, we do not feel justified in proposing a new species on the information so far available.

Aeromonas hydrophila. The four strains of the Aeromonas hydrophila phenon include the neotype A. hydrophila subsp. hydrophila no. 64
TABLE 3. DNA base composition of selected $V$. metschnikovii and group $F$ strains

\begin{tabular}{cc}
\hline \multicolumn{1}{c}{ Strain } & Mol\% G+C \\
\hline V. metschnikovii & \\
1 Lectotype NCTC 8443 & 45.4 \\
3 & 45.6 \\
4 & 44.9 \\
5 NCTC 8564 & 45.4 \\
11NCTC 11171 & 45.9 \\
18 NCTC 11172 & 45.4 \\
19 & 46.9 \\
31 & 46.9 \\
38 NCTC 11170 & 44.2 \\
Group F & \\
57 & 52.0 \\
60 & 52.2 \\
\hline
\end{tabular}

a The mole percent $\mathrm{G}+\mathrm{C}$ of a similar strain isolated from a patient with diarrhea was 51.5.

(NCTC 8049), and all the strains conform to the description of Schubert (22).

Taxonomy and nomenclature of $V$. metschnikovii. The formation of separate clusters corresponding to $V$. metschnikovii, $V$. proteus, and the wild strains could be interpreted as an indication of separate strains or biotypes. However, two of the $V$. proteus strains and two of the $V$. metschnikovii strains are supposedly the same organism received from two sources, in each case one from the American Type Culture. Collection or the National Collection of Type Cultures (NCTC) and the other via R. Hugh. None of these strains was as highly related to one another as would have been expected. Furthermore, all of the strains falling in the clusters corresponding to $V$. metschnikovii and V. proteus are culture collection strains that, having been subcultured over many years, may have changed. We believe this has happened with certain old NCTC strains of $V$. cholerae, which differ from strains isolated more recently in being less versatile, growing less rapidly, and being unable to grow on TCBS. It is possible that $V$. proteus and $V$. metschnikovii strains may have "changed" in a similar way. We have not yet discovered any wild isolate that matches the culture collection strains exactly. In the case of $V$. metschnikovii, this may be because they are unable to grow on TCBS, but we do have a few fresh isolates, such as strain 20 , that are unable to grow on TCBS and otherwise match the other wild strains. We therefore do not believe we are justified in dividing the oxidasenegative group into separate species or biotypes at the moment. We think they should be included in a single species.

Although these strains are all oxidase negative and unable to reduce nitrate to nitrite or trimethylamine oxide to trimethylamine, they are 
otherwise similar to the other species of the genus Vibrio. The DNA base composition of the nine strains examined was in the range 44.2 to $46.9 \mathrm{~mol} \% \mathrm{G}+\mathrm{C}$, with a mean of $45.6 \mathrm{~mol} \%$ $\mathrm{G}+\mathrm{C}$ (Table 3). This is within the range for the genus Vibrio (40 to $50 \mathrm{~mol} \%$ ) and too low for the genus Aeromonas ( 57 to $63 \mathrm{~mol} \%$ ). We do not think that they are closely related to the genus Photobacterium, since they do not produce gas, are nonluminescent, and possess amylase and gelatinase. The two species in our study with which they have greatest similarity are $V$. cholerae and $V$. anguillarum. Thus, there is insufficient evidence to create a new genus for this oxidase-negative group of organisms; they should be retained within the genus Vibrio.

The $V$. cholerae phenon does not link with the oxidase-negative phenon until the $85 \%$ level of similarity. There are also several consistent differences between strains of the two phenons, notably decarboxylase reactions, salt requirement for growth, and acid production from inositol and lactose. Therefore, we cannot support the suggestion of Shewan and Véron (23) that this group be considered as a biotype of $V$. cholerae.

According to the Index Bergeyana (2) V.proteus Buchner is an illegitimate name. From the records of the NCTC (Lapage, personal communication, 1977), V. metschnikovii M34, NCTC 8443 (our computer number 1), is the same as the strain listed as NCTC 262 in the first NCTC catalog of 1922. In this it says "originally isolated by Gamaleia from disease of fowls." Accordingly, we conclude that this phenon should be named $V$. metschnikovii and that the lectotype is NCTC 8443 . Since this strain has been in culture collections for many years and differs from the wild strains in certain characteristics (Table 2), we suggest that our strain 38 (NCTC 11170), isolated from cockles, may be used as a working strain. All of the $V$. metschnikovii strains in our study were polarly flagellated, gram-negative, asporogenous, slightly curved rods. Electron micrographs of the lectotype and strains with computer numbers 11,18 , and 38 are shown in Fig. 3. The inclusion of these organisms in the genus Vibrio would require its definition to be changed accordingly. We also suggest that the minimal characters for the identification of vibrio species recommended by Hugh and Sakazaki (11) be emended as shown in Table 4.

Vibrio metschnikovii Gamaleia 1888 (synonyms Vibrio proteus Buchner 1885; Vibrio cholerae biotype proteus Shewan and Veron 1974). Named for E. Metschnikoff, a Russian bacteriologist.

Description of species. Short rods; axis straight or curved; sides parallel; ends rounded; occurring singly, in pairs, and occasionally in short chains of three to four organisms; pleo-

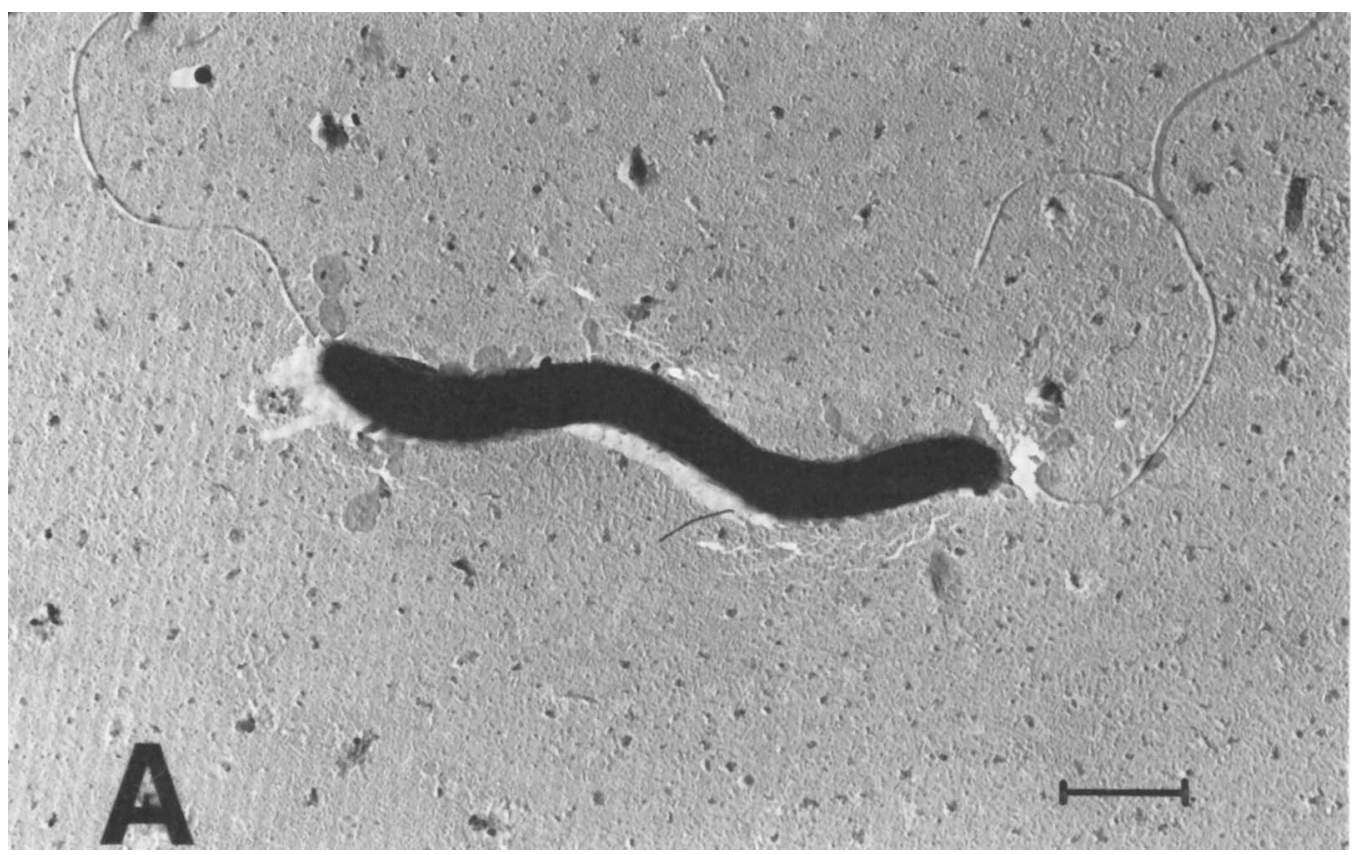

FIG. 3. Electron micrographs of V. metschnikovii strains. (A) Lectotype strain 1, NCTC 8443; (B) strain 38; (C) strain 11; (D) strain 18. Bars indicate $1 \mu \mathrm{m}$. 

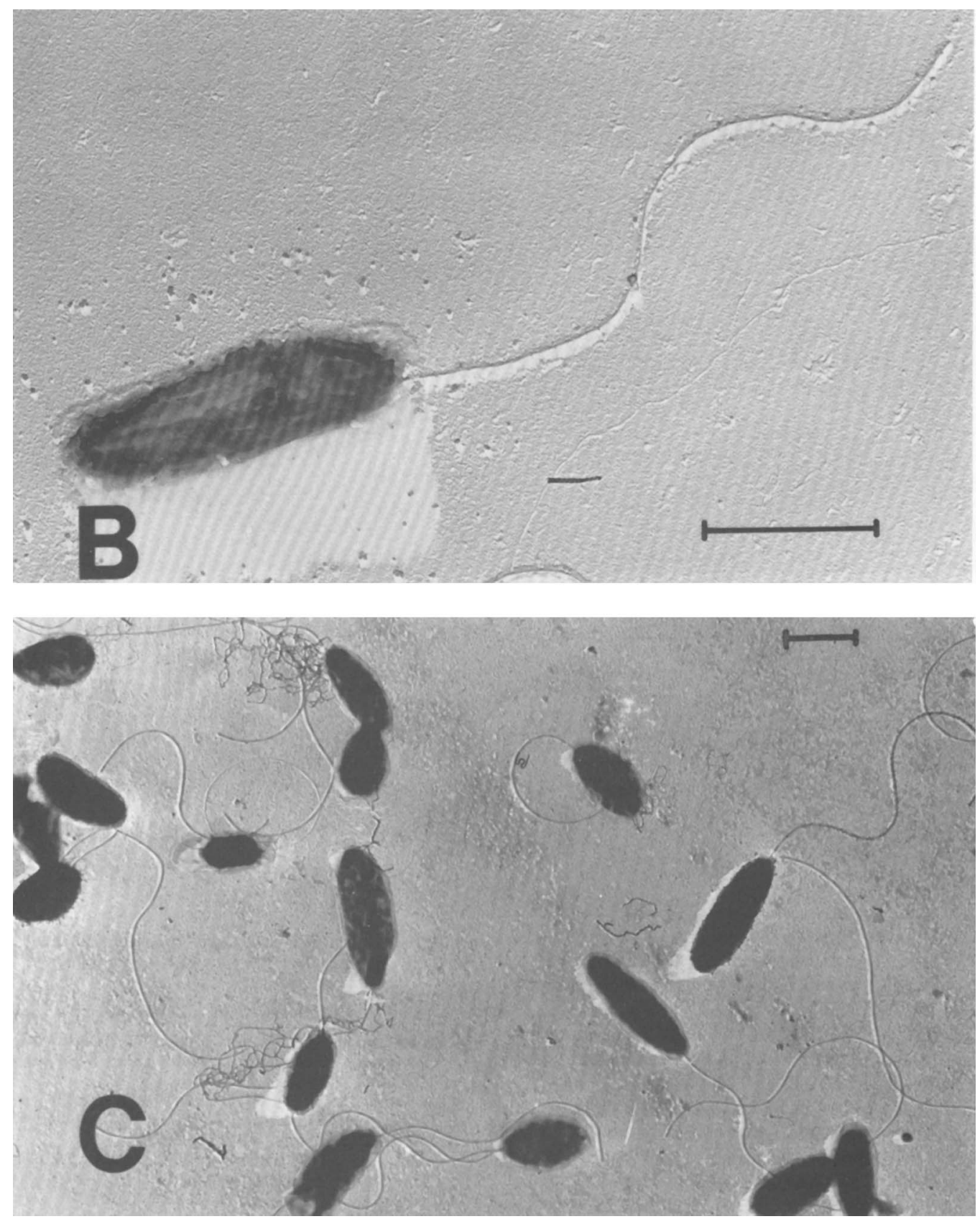

Figs. $3 B-C$

morphic; 0.5 by 1.5 to $2.5 \mu \mathrm{m}$. Motile by means of a single polar flagellum. Sodium chloride is required for growth, but no organic growth factors are required. The biochemical and physiological characteristics of the species and lectotype are shown in Table 2 . Those characteristics particularly useful for the identification of the species are summarized in Table 5.

Habitat: Very widely distributed throughout the aquatic environment, particularly in rivers, estuaries, and sewage. Sometimes isolated from animal intestines, including that of humans, but there is no evidence to suggest they cause enteritis in humans or animals. Frequently isolated 


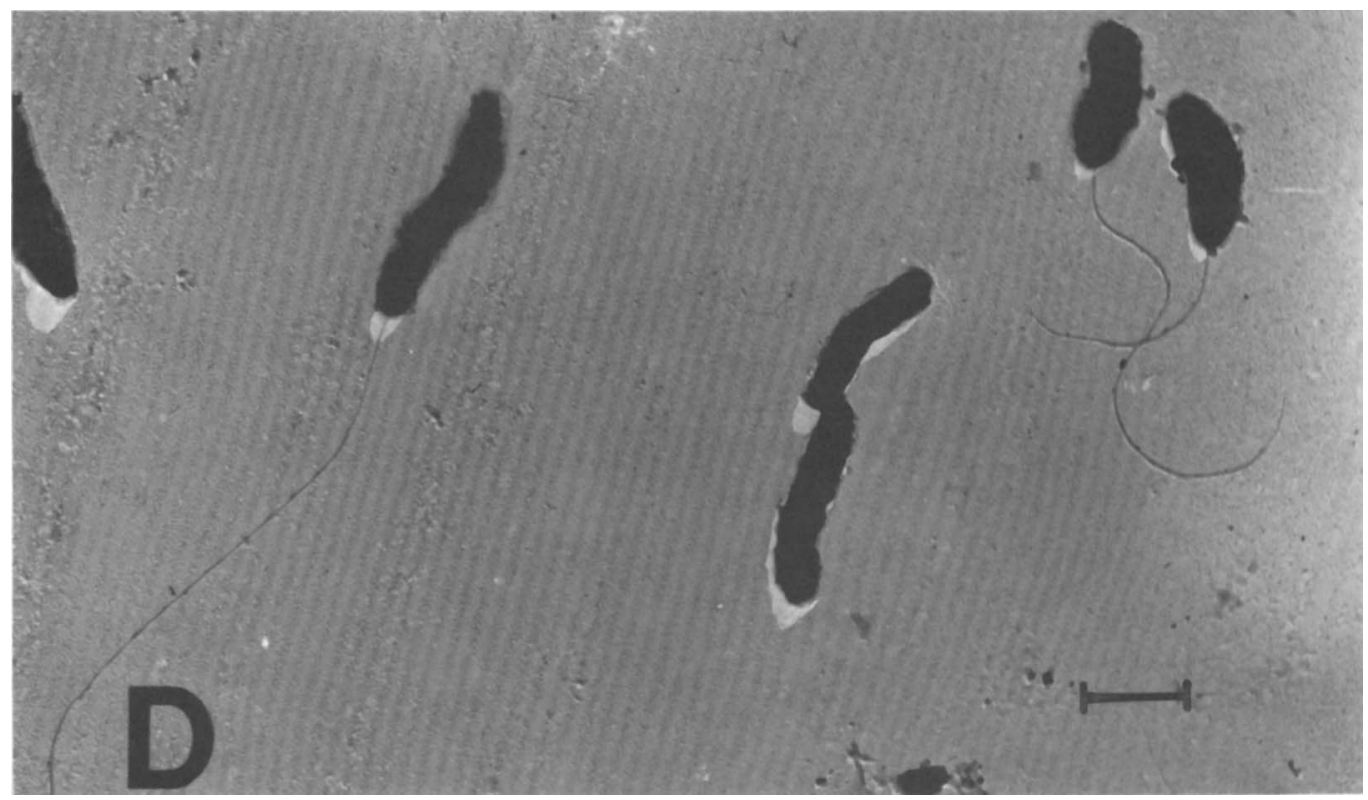

Fig. $3 D$

TABLE 4. Characters useful for the identification of Vibrio species

\begin{tabular}{|c|c|}
\hline Character & Results \\
\hline \multicolumn{2}{|l|}{ Polar flagellum } \\
\hline enous rod & \\
\hline Indor & $t^{a}$ \\
\hline oleum seal & + \\
\hline Glucose, gas & - \\
\hline $\mathbf{D}-\mathbf{M}$ & + \\
\hline $40-50 \mathrm{~mol} \% \mathrm{G}+\mathrm{C}$ & \\
\hline $0 / 129$ (150- $\mu \mathrm{g}$ disk) susceptible & + \\
\hline Extracellular deoxyribo & + \\
\hline
\end{tabular}

${ }^{a}$ Except V. metschnikovii.

from various shellfish, e.g., cockles, clams, oysters, and lobsters.

The $\mathrm{G}+\mathrm{C}$ content of the DNA is in the range 42.5 to $46.9 \mathrm{~mol} \%$ for the 9 strains examined (Table 3).

The lectotype is NCTC 8443. Since this strain has been in culture collections for many years and differs from the fresh isolates in certain characters (Table 2), we have also deposited strains MV2339, MV2366, and MV2376 in the NCTC under the numbers NCTC 11171, NCTC 11172 , and NCTC 11173 , respectively. We recommend strain MV2484 (NCTC 11170), originally isolated from cockles, as a working strain.

\section{ACKNOWLEDGMENTS}

We thank W. R. Willcox of the NCTC for performing the computer analysis; S. P. Lapage, curator of the NCTC, for his advice and permitting us to use the NCTC computer facilities; D. M. Gibson of Torry Research Station, Aberdeen,
TABLE 5. Characters useful for the identification of $V$. metschnikovii

\begin{tabular}{|c|c|}
\hline Character & Result \\
\hline Oxidase & - \\
\hline Nitrate reduction & - \\
\hline Arginine dihydrolase & + \\
\hline Lysine decarboxylase & $\mathrm{V}^{a}$ \\
\hline Ornithine decarboxylase & - \\
\hline $0 / 129(10 \mu \mathrm{g}$ disk) susceptible & $\ldots+$ \\
\hline Sucrose . . . . . . & $\ldots+$ \\
\hline
\end{tabular}

${ }^{a}$ Variable reaction.

Scotland, for determining the DNA base compositions, and W. Hodgkiss, also of Torry, for the electron micrographs.

\section{UTTERATURE CITED}

1. Baumann, P., L. Baumann, and M. Mandel. 1971. Taxonomy of marine bacteria: the genus Beneckea. J. Bacteriol. 107:268-294.

2. Buchanan, R. E., J. G. Holt, and E. F. Lessel (ed.). 1966. Index bergeyana, p. 1472. The Williams and Wilkins Co., Baltimore.

3. Buchner, H. 1885. Über die Koch'schen und FinklerPrior'schen "Kommabacillen" Sitzungersber Gesel Morphol. Physiol. München 1:1-10.

4. Colwell, R. R. 1970. Polyphasic taxonomy of the genus Vibrio: numerical taxonomy of Vibrio cholerae, Vibrio parahaemolyticus, and related Vibrio species. 3. Bacteriol. 104:410-433.

5. Cowan, S. T. 1974. Cowan \& Steel's manual for the identification of medical bacteria, 2nd ed. Cambridge University Press, Cambridge.

6. Crosby, N. T. 1967. The determination of nitrite in water using Cleve's acid, 1-naphthylamine-7-sulphonic acid. J. Soc. Water Treat. Exam. 16:51-55.

7. Finkelstein, R. A., and S. Mukerjee. 1963. Haemagglutination: a rapid method for differentiating Vibrio chol- 
erae and El Tor vibrios. Proc. Soc. Exp. Biol. Med. 112:355-359.

8. Gamleia, M. N. 1888. Vibrio metschnikovii (n.sp.) et ses raports avec le microbe du choléra asiatique. Ann. Inst. Pasteur (Paris). 2:482-488.

9. Gauthier, M. J. J. M. Shewan, D. M. Gibson, and J. V. Lee. 1975. Taxonomic position and seasonal variations in marine neritic environment of some gram-negative antibiotic-producing bacteria. J. Gen. Microbiol. 87:211-218.

10. Hugh, R., and E. Leifson. 1953. The taxonomic significance of fermentative versus oxidative metabolism of carbohydrates by various gram-negative bacteria. J. Bacteriol. 66:24-26.

11. Hugh, R., and R. Sakazaki. 1972. Minimal number of characters for the identification of Vibrio species, Vibrio cholerae and Vibrio parahaemolyticus. J. Conf. Public Health Lab. Directors 30:133-137.

12. International Committee on Nomenclature of Bacteria, Subcommittee on the Taxonomy of Vibrios. 1975. Minutes of the closed meeting, 3 September 1974. Int. J. Syst. Bacteriol. 25:389-391.

13. International Committee on Systematic Bacteriology, Subcommittee on Taxonomy of Vibrios. 1972. Report (1966-1970) of the Subcommittee on Taxonomy of Vibrios to the International Committee on Nomenclature of Bacteria. Int. J. Syst. Bacteriol. 22:123.

14. Kovács, N. 1956. Identification of Pseudomonas pyocyanea by the oxidase reaction. Nature (London) 178:703.

15. Laycock, R. H., and L. W. Regier. 1971. Trimethylamine-producing bacteria on haddock (Melanogrammus aeglefinus) fillets during refrigerated storage. J. Fish. Res. Board Can. 28:305-309.

16. Marmur, J. 1961. A procedure for the isolation of deoxyribonucleic acid from microorganisms. J. Mol. Biol.
3:208-218.

17. Marmur, J., and P. Doty. 1962. Determination of the base composition of deoxyribonucleic acid from its thermal denaturation temperature. J. Mol. Biol. 5:109-118.

18. Murray, C. K., and D. M. Gibson. 1972. An investigation of the method of determining trimethylamine in fish muscle extracts by the formation of its picrate salt: part 1. J. Food. Technol. 7:34-46.

19. Rhodes, M. E. 1958. The cytology of Pseudomonas spp. as revealed by silver-plating staining method. J. Gen. Microbiol. 18:639-648.

20. Sakazaki, R., K. Tamura, K. Ikuta, and M. Sebald. 1970. Taxonomical studies on marine vibrios, $p$ 583-593. In H. Iizuka and T. Hasegawa (ed.), Proceedings of the International Conference on Culture Collections, Tokyo, 1968. University Park Press, Baltimore.

21. Sakazaki, R., K. Tamura, and M. Murase. 1971. Determination of the hemolytic activity of Vibrio cholerae. Jpn. J. Med. Sci. Biol. 24:83-91.

22. Schubert, R. H. W. 1974. Genus II. Aeromonas Kluyver and van Niel 1936, 398, p. 345-348. In R. E. Buchanan and N. E. Gibbons (ed.), Bergey's manual of determinative bacteriology, 8 th ed. The Williams and Wilkins Co., Baltimore.

23. Shewan, J. M., and M. V́éron. 1974. Genus I. Vibrio Pacini 1854, 411, p. 340-345. In R. E. Buchanan and N. E. Gibbons (ed.), Bergey's manual of determinative bacteriology, 8th ed. The Williams and Wilkins Co. Baltimore.

24. Sneath, P. H. A., and R. R. Sokal. 1973. Numerical taxonomy: the principles and practice of numerical classification. W. H. Freeman, San Francisco.

25. Thornley, M. J. 1960. The differentiation of Pseudomonas from other gram-negative bacteria on the basis of arginine metabolism. J. Appl. Bacteriol. 23:23-52. 